\title{
Research on Development and Test Analysis of Full-Scale Fatigue Test System of X65 Submarine Pipeline
}

\author{
Yanhua $\mathrm{Hu}^{1, \mathrm{a}}$,Yukun Wang ${ }^{2, \mathrm{~b}}$,Pengyu Jia ${ }^{3, \mathrm{c}, \mathrm{c}}$ Jianyu $\mathrm{Lv}^{4 *, \mathrm{~d}}$,Mingchao Wang ${ }^{5, \mathrm{e}}$ \\ ${ }^{1}$ Tianjin Research Institute for Water Transport Engineering, M.O.T Tianjin, China \\ ${ }^{2}$ Tianjin Dongfang Tairui Technology CO. LTD. Tianjin, China \\ ${ }^{3}$ Tianjin Research Institute for Water Transport Engineering, M.O.T. Tianjin, China \\ ${ }^{4}$ Tianjin Research Institute for Water Transport Engineering, M.O.T. Tianjin, China \\ ${ }^{5}$ Tianjin Research Institute for Water Transport Engineering, M.O.T. Tianjin, China
}

\begin{abstract}
The fatigue performance of welded joints of submarine pipelines is directly related to the safety and economic benefits of welded structures. Considering the limitations of fatigue calculation, anti-fatigue design and small-scale fatigue test in the evaluation and analysis of pipeline fatigue life, this paper demonstrated the feasibility, scientificity and advancement of submarine pipeline full-scale fatigue test technology in engineering applications. Consequently, a full-scale fatigue test system and its test analysis technology applied for ZY-PFS2000 pipelines have been first developed in China, in which the effects of welding residual stress, stress concentration, initial welding defects, pipeline internal pressure shutdown and internal medium fluctuations on the fatigue life of full-scale pipelines were comprehensively taken into account. Through the full-scale fatigue test (four-point bending + internal pressure) of the X65 submarine pipeline, the fatigue cycles of different specifications of pipelines under different stress amplitudes were obtained. Moreover, the fatigue loading results were evaluated and analyzed in accordance with the international standard of BS 7608 and DNV C203. The research in this paper is conducive to accumulating full-scale fatigue performance data for submarine pipelines in China, not only offering a quantitative basis for the subsequent full-scale fatigue life evaluation and the safety operation cycle, but also providing a reference direction for the future development of submarine pipeline full-scale fatigue test technology.
\end{abstract}

\section{Introduction}

With the increase in offshore oil development, due to the complex and particular submarine environment and offshore production, the safety of submarine pipelines during service has become a special concern during their design, laying, and operation. Among the many factors that cause submarine pipeline accidents, fatigue damage is the main reason for the failure of submarine pipelines [13]. The fatigue damage of submarine pipelines is often caused by various alternating stresses in the pipeline [4,5]. The alternating stress expands the internal and surface defects of the pipeline, and finally causes the fatigue fracture of the pipeline, resulting in the serious consequence of forcing the interruption of the oil and gas supply.

The main feature of the welded structure of the submarine pipeline is that there are welding residual stresses, various welding defects, and stress concentration at the welded joints. Tests have proved [6] that the controlling factor for the initiation and propagation of fatigue cracks was not the traditionally believed stress ratio and maximum stress, but the stress amplitude, that is, the difference between the maximum stress and the minimum stress. The residual stress and stress concentration factors at the welded joints of different welded structures are different, so the relationship between the stress amplitude and the number of failure cycles under each structure is different. The same magnitude of stress amplitude acts on different welded structures, so the number of stress cycles it can withstand is also different, that is, the fatigue life is different $[7,8]$. Because the welded structure still has initial welding defects, it is likely to develop fatigue cracks, which is the main reason for the fatigue failure of submarine pipelines [9]. Therefore, in the full-scale fatigue test of submarine pipelines, it is necessary to focus on the influence of welding residual stress and stress concentration factor on pipeline fatigue life.

To ensure the safety and stable operation of submarine pipelines, it is necessary to analyze their fatigue performance [10-14]. At present, the fatigue research of submarine pipelines at home and abroad is developing rapidly, and many research results have been obtained. In terms of fatigue calculation and anti-fatigue design, methods such as nominal stress fatigue design, local stress-strain analysis, damage tolerance design, and fatigue reliability design have been formed. However, because fatigue design can only approximate the fatigue

\footnotetext{
ahuyanhua@tk-aq.com b bangyukun@tk-aq.com

cjiapengyu@tk-aq.com d d* Corresponding author: lvjianyu@tk-aq.com

ewangmingchao@tk-aq.com
} 
life of pipelines, fatigue test methods are often used in engineering applications to evaluate the fatigue life of pipelines $[15,16]$. However, because the fatigue design method can only approximate the fatigue life of pipelines, the fatigue test method is often used instead in engineering applications to evaluate the fatigue life of pipelines $[15,16]$.

In the past, the pipeline fatigue test used to adopt the small-scale fatigue test analysis method. This method not only ignores the size effect in the test process but also releases the influence of welding residual stress and stress concentration during the specimen processing, which leads to high test results. In this way, the results need to be adjusted and corrected appropriately in actual applications. The rapid growth of the electro-hydraulic servo fatigue testing machine has driven the research focus of foreign countries from small-scale to full-scale fatigue tests in recent years, and certain research results have been derived and applied in submarine key pipeline engineering $[17,18]$. It can be confirmed that the test data obtained from the full-scale fatigue test of the pipeline can be used for its life prediction and safety evaluation, which has gradually become the consensus of the industry.

A full-scale fatigue test system and its test analysis technology applied for ZY-PFS2000 pipelines are developed, in which the effects of welding residual stress, stress concentration, initial welding defects, pipeline internal pressure shutdown, and internal medium fluctuations on the fatigue life of full-scale pipelines are comprehensively taken into account. Through the fullscale fatigue test (four-point bending + internal pressure) of the X65 submarine pipeline, the fatigue cycles of different specifications of pipelines under different stress amplitudes are obtained. Moreover, the fatigue loading results are evaluated and analyzed under the international standard of BS 7608 and DNV C203.

The rest of this paper is structured as follows. The second part summarizes the research on the full-scale fatigue test technology of submarine pipelines. The third part introduces case studies and discussions, including the system configuration of the ZY-PFS2000 full-scale pipeline fatigue testing machine its performance parameters, and the comparative analysis of the experimental results. The fourth part summarizes the research results of this paper and discusses the limitations and future research directions.

\section{Literature Review}

The full-scale fatigue test technology of submarine pipelines is time-consuming, expensive, and technically difficult $[19,20]$, and requires a high performance for fullscale fatigue equipment, so the progress of research in the 1990s was once hindered. In recent years, the rapid development of electronic computer-controlled electrohydraulic servo fatigue test technology has driven the research progress of full-scale fatigue test of pipelines, and some technical breakthroughs have been made. At present, some developed countries in Europe and the United States have used full-scale fatigue test data to evaluate the fatigue life of submarine pipelines in submarine oil and gas pipeline laying projects and carried out anti-fatigue design calculations on the pipeline relying on the S-N curve obtained from the full-scale fatigue test, all of which are contributing to the promotion of the pipeline full-scale fatigue test technology.

Early in the late 1990s, the British Welding Institute conducted a lot of research on the fatigue reliability and safety evaluation of submarine pipelines. They applied their technological achievements to submarine oil and gas pipeline laying projects, which laid a reference foundation for other countries to develop pipeline full-scale fatigue test technology. Stephen J Maddox et al. [21,22] conducted an experimental study on the full-scale fatigue life of submarine pipeline welded joints based on the pipeline fatigue design curve, focusing on the influence of different welding process methods (under tensile load) on the fatigue life of welded joints. It was notable that Stephen J Maddox et al. carried out the first full-scale fatigue test technology research for the welding technology of submarine pipeline welded joints. They quantitatively demonstrated the ideal value range of various factors from the experimental methods, including the unfitness and the excess weld metal of the welded joint, and pioneered research ideas for improving the fatigue performance of submarine pipelines during service.

To evaluate the structural integrity and fatigue performance of nuclear power plant piping systems (material of ASTM A106B and ASTM A333 Gr.6, pipe diameter of $\varphi 114.3 \mathrm{~mm}$, wall thickness of $8.6 \mathrm{~mm}$, pipe internal pressure of 10.9 MPa), Kunio Hasegawa et al. [23] of Hitachi, Japan, conducted a low-cycle full-scale fatigue test on steel pipes with prefabricated defects under ambient temperature conditions. The test adopted a selfdesigned full-scale fatigue testing machine, mainly composed of load rollers, load brackets, and fixed benches. It can complete full-scale fatigue tests for small-diameter pipes (pipe diameter of $\varphi 114.3 \mathrm{~mm}$ ) with pipe lengths within the range of $300 \sim 960 \mathrm{~mm}$. The low-cycle fatigue strength curves and cyclic stress-strain curves of the prefabricated defective pipelines and the complete pipelines were compared in the test. The comparison showed that prefabricated defects had not affected the structural integrity of the pipeline, and the pipeline was damaged within the range of the stress and cycles caused by the earthquake. Owing to the welding residual stress at the welded joints, the high-cycle full-scale fatigue life of the pipeline was lower than the requirements of the fatigue design curve in the third part of the ASME standard. This indicated that welding defects had a great impact on the pipeline fatigue performance, especially in the high-cycle fatigue zone where crack initiation accounts for a large part of the fatigue life. In brief, this research was the first to study the influence of fatigue crack prefabrication on the pipeline fatigue life through a full-scale fatigue test. Apart from overcoming the shortcomings of low loading frequency and long test period in the conventional fourpoint bending fatigue testing machine, it laid a reference foundation for the study of fatigue crack prefabrication and its propagation mechanism in subsequent full-scale fatigue tests.

G. F. Miscow et al. [24] designed and developed a set of full-scale fatigue test equipment and a supporting 
pipeline fatigue simulator to evaluate the full-scale fatigue test of submarine drill pipes. The test principle was similar to the small-scale rotating bending test, where the pipe was equal to a simply supported beam with the weld in the middle of the beam. Through two identical loading devices, the transverse load was loaded at two points equidistant from the end, so that a certain equivalent bending moment was aroused at the middle section of the specimen, that is, the girth weld. When the loading device input the transverse load of the alternating cycle, the source of fatigue cracks occurred at the welded joint under the influence of welding residual stress and stress concentration, eventually resulting in the fatigue failure.

In addition, T. A. Netto et al. [25] further studied the local deformation mechanism of pre-strained welded joints with defects under bending load. The study results indicated that highly localized strain may be generated at the tip with defects, depending on the type (incomplete fusion or penetration) and size of the defect. They first obtained the fatigue life of pre-strained joints with different defects through a full-scale fatigue test. Following the finite element analysis model and a specific algorithm based on linear fracture mechanics, the material property changes due to pre-strain were explained and finally used to evaluate the fatigue life of a typical joint, providing a reference for the technical development of fatigue performance evaluation in the deep water standpipe welding standard.

In recent years, Norway has made many research results in the field of submarine pipeline fatigue integrity evaluation. In particular, full-scale fatigue test studies were conducted on the fatigue life of submarine pipelines, such as casing pipes, standpipes, and drill pipes, to ensure the safe operation of submarine oil and gas pipelines. It was reported [26] that the large fatigue testing machine used in Norway was mainly composed of the hydraulic power system, a hydraulic servo actuator for static and dynamic tests, control system, operating system, and dynamic actuator with a maximum loading capacity of $2000 \mathrm{KN}$. The equipment had agile and operation-oriented loading procedures including random load sequence and variable signal bandwidth. It can perform full-scale fatigue tests in the submarine environment with temperature control and cathodic protection, as well as full-scale simulation tests for large structures with automatic crack propagation monitoring. So far, the system has completed full-scale fatigue tests of various pipelines and various types of welded joints and has accumulated valuable full-scale fatigue test data and fatigue design data for pipelines.

As the development of full-scale fatigue test technology abroad has become increasingly mature, China has also begun to gradually develop full-scale fatigue test technology for pipelines due to its requirements for pipeline fatigue safety evaluation, and some research results have been obtained. In terms of the exploration and research on the full-scale fatigue analysis of submarine pipelines, Li et al. [27] from CNPC Tubular Goods Research Institute firstly carried out the internal pressure full-scale fatigue test of physical steel pipes in China and conducted the evaluation, prediction, and verification of the remaining pipeline life based on the test data of the crack propagation rate of the small-scale fatigue specimen. Based on the above research, Zhuang et al. [28] carried out a full-scale internal pressure fatigue test for X60 spiral submerged arc welded pipes and obtained the influence of internal pressure fluctuations on pipeline fatigue. Moreover, under the premise of ignoring the early fatigue crack initiation life of the pipeline, the remaining pipeline life is quantitatively evaluated based on the fatigue crack propagation rate derived from the small-scale fatigue test. The above research firstly introduced the concept of fullscale fatigue and fatigue crack prefabrication in China, which played a positive role in promoting the advancement of domestic full-scale fatigue test technology.

Through analysis of the above-mentioned domestic and foreign pipeline full-scale fatigue test technology, it is found that: (1) The loading test range of foreign full-scale fatigue test machines is limited, and the maximum loading diameter is within $\varphi 650 \mathrm{~mm}$; (2) The full-scale fatigue test equipment is a mainly horizontal structure, and the purpose is to increase the system stroke to meet the fullscale requirements of the pipeline; (3) The test type is mainly bending test (including rotating bending and fourpoint bending), supplemented by axial tensile fatigue test and internal pressure fatigue test; (4) The analysis focuses on fatigue life evaluation of full-scale fatigue welded joints are not consistent in various countries, but the influence of factors including residual stress, stress concentration, fatigue initial defects, and welding process are mainly considered. Therefore, when carrying out fullscale fatigue test technology research in China, on one hand, it is necessary to absorb and utilize existing foreign technology achievements and establish a domestic test analysis system as soon as possible; on the other hand, despite the reference to foreign technical foundations, it is required to form a full-scale fatigue test technology with Chinese characteristics through full-scale fatigue equipment and innovative fatigue test theory system.

\section{Full-scale fatigue test system of ZY- PFS2000 pipelines and its test analysis technology}

\subsection{System composition}

Based on domestic and foreign research on full-scale fatigue test technology, China has developed the ZYPFS2000 full-scale pipeline fatigue test system. The ZYPFS2000 full-scale pipeline fatigue test system can be used to simulate the alternating stress of various types of full-scale pipelines during service. Apart from the pipeline fatigue performance in its external variable loads such as wave load and current load, it can also simulate the influence of the pressure fluctuation of the conveying medium inside the pipeline on its fatigue life, so that it is used to evaluate the fatigue performance and fatigue life of various types of full-scale pipelines. 


\subsection{Mechanical loading unit}

During the test, the mechanical loading unit can adjust the size of the fixture outside the pipeline, the span of the actuator, the length, and the value of the equivalent bending moment of the welded joint. At the same time, by simulating the input form of the external load, it can adjust the parameters of the external load in real-time, such as input waveform, load amplitude, phase angle, loading frequency. As a result, the requirements for the test parameters of the test pipeline in the full-scale fatigue test are met, including external load, bending moment, stress amplitude, and load spacing, hereby realizing the coordinated or separate dynamic and static loading of the three-point and four-point bending of the test pipeline. The main technical parameters of the mechanical loading unit are shown in Table 1.

Table1. Main technical parameters

\begin{tabular}{|c|c|c|c|c|c|}
\hline $\begin{array}{c}\text { Rated load } \\
\text { of each } \\
\text { actuator }\end{array}$ & $\begin{array}{c}\text { Stroke of } \\
\text { the } \\
\text { bending } \\
\text { servo } \\
\text { actuator }\end{array}$ & $\begin{array}{c}\text { System } \\
\text { static } \\
\text { accuracy }\end{array}$ & $\begin{array}{c}\text { System } \\
\text { dynamic } \\
\text { accuracy }\end{array}$ & $\begin{array}{c}\text { System } \\
\text { operating } \\
\text { frequency } \\
\text { range }\end{array}$ & $\begin{array}{c}\text { Maximu } \\
\text { m } \\
\text { internal } \\
\text { pressure } \\
\text { of test } \\
\text { pipeline }\end{array}$ \\
\hline $\begin{array}{c}1000 \mathrm{KN} ; \\
\text { Divide by } \\
\text { attenuation } \\
\text { multiples of } \\
1 \text { and } 10\end{array}$ & $\pm 125 \mathrm{~mm}$ & $\leqslant \pm 1 \%$ & $\leqslant \pm 3 \%$ & $0.01 \mathrm{~Hz} \sim 5 \mathrm{~Hz}$ & $21 \mathrm{MPa}$ \\
\hline
\end{tabular}

\subsection{Hydraulic loading unit}

The hydraulic loading unit is mainly composed of an oil tank, a hydraulic pump set, an overflow valve, a highprecision oil filter, a cooler, a control valve, and a power distribution box. The core component of the hydraulic pump unit adopts a combination of meshing gear pump and motor. On account of the requirements of the system loading amplitude, loading frequency, and actuator stroke, the output flow rate is finally determined to be $500 \mathrm{~L} / \mathrm{min}$ based on the functions and technical indicators of the fullscale fatigue test system. In addition to realizing ondemand distribution, real-time startup, and dynamic control of system power sources, it can also meet the dynamic response requirements of multi-functional pipeline fatigue tests, including pipeline static loading, dynamic loading, and internal pressure loading. Besides, the hydraulic unit cooperates with servo control valves, high-precision oil filters, accumulators, and coolers to achieve protection functions (such as low liquid level, over-temperature, blocked filter, overload alarm, and automatic pressure relief and oil return) to ensure the safe, stable and efficient operation of the system power source.

\subsection{Control unit}

The core of the control unit is an all-digital measurement and controlling device composed of 240 series DSP devices from the TI Company, which enables to run as fast as 0.05 microseconds per instruction. The function of the measurement and controlling device is realized by an alldigital measurement and controlling board card, which is in line with the form of the ISA bus standard circuit board and installed in the slot of the industrial control computer. The all-digital measurement and controlling circuit board integrates displacement amplifier, load amplifier, deformation amplifier (optional), servo drive, and DSP controller. It adopts dual-port RAM and large-scale programmable device CPLD to make the system highly integrated.

The computer soft control panel consists of the main menu and two main control panels. During the test, the mechanical loading unit utilizes three servo actuators with low damping and high response, in which the remote control and operation of the two load servo actuators can be realized through two main control panels, and the remote control of the internal pressure servo actuators can be realized through the main menu. After the command is input, the feedback signals of the electro-hydraulic servo valve and pressure sensor are collected to perform differential closed-loop servo control of the test load and pressure. It has many advantages, such as fast response speed, high control accuracy, wide bandwidth, and many types of test waveforms, indicating that the performance and structure of the system are superior to the domestic conventional fatigue test control system. The control algorithm adopts the typical digital PID position control algorithm, which can perform remote control of pressure increase, pressure maintenance, pressure reduction, stepping, amplitude stabilization, and alarm triggering during the test. What's more, various physical tests such as static test, steady amplitude fatigue, internal pressure fatigue, and random fatigue spectrum are becoming tangible.

\subsection{Full-scale fatigue test analysis technology}

Many key technical problems are firstly solved in the test including full-scale fatigue crack prefabrication, fatigue load correction and application, as well as load spectrum compilation and expansion, in which the effects of welding residual stress, stress concentration, initial welding defects are comprehensively taken into account. Moreover, a series of proprietary technologies are invented, including full-scale fatigue testing, stress-strain testing, fatigue crack detection, multi-type stress superposition analysis of pipelines, and fatigue performance evaluation.

The following technologies, on the one hand, are beneficial to increasing engineering personnel's understanding of the fatigue resistance of welded structures, and improve the design capabilities of pipeline structure forms and joint forms, which enables the designed welding structure to be more rational and develop higher fatigue strength while strictly controlling the welding quality. On the other hand, these technologies directly face the weakness of poor fatigue performance of welded joints, assisting in strict control of welding quality during the manufacturing process to prevent and reduce the occurrence of welding defects. What's more, it is necessary to take effective processing measures after the completion of the welded joint and during the use process to further improve the fatigue strength of the joint, thereby increasing its ability to withstand dynamic loads and 
extending its service life.

\section{Full-scale fatigue test results and analysis of submarine pipelines}

So far, the full-scale fatigue tests with simultaneous loading of four-point bending and internal pressure fatigue of X65 pipelines have been completed on the ZY-PFS2000 full-scale pipeline fatigue testing machine within the diameters of $\varphi 108, \varphi 323.9$, and $\varphi 610$. The loading range of the welded joint is $124 \mathrm{MPa} \sim 298 \mathrm{MPa}$, and the internal pressure loading range of the pipeline is $0 \sim 20 \mathrm{MPa}$. As shown in Figure 5, the fatigue life of joints under different stress ranges is learned, and the tests are evaluated and analyzed by the international standard of BS 7608 and DNV C203.

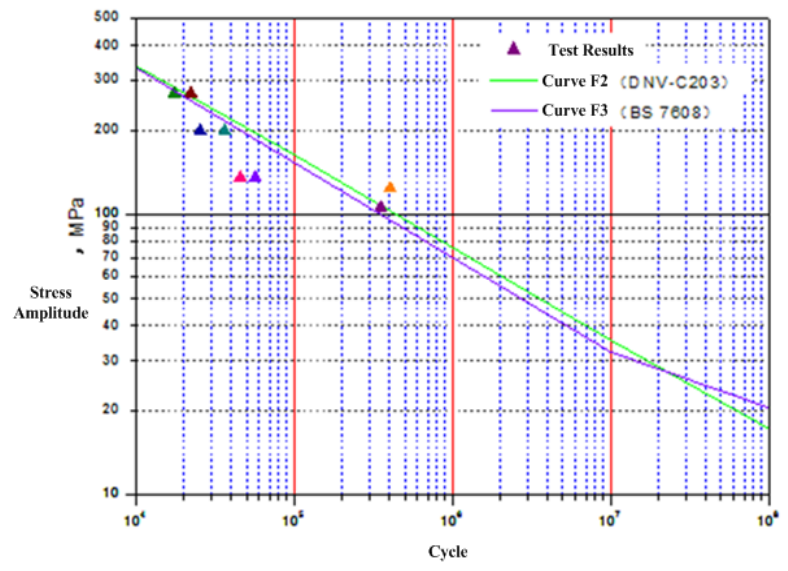

Figure1. All-digital measurement and controlling device

As is shown in Figure 1 and Figure 2, the failure modes of pipeline fatigue can be classified into three types: 1) the location of the pipeline failure is unknown; 2) the location of the pipeline failure is the base material; 3) the location of the pipeline failure is the welded joint. Combined with the schematic diagram of on-site pipeline failure, the following conclusions can be drawn:

\subsection{For pipelines whose failure location is unknown}

The fatigue cycle of X65 submarine welded joints has reached the standard qualification requirements, and the test should be stopped due to time considerations. In this case, it is deemed that the fatigue life of the pipeline meets the requirements in the assessment.

\subsection{For pipelines whose failure location is the base material}

(1) In general, fatigue cracks originate in the contact area (base material) between the pipeline and the fixture, first penetrate the wall thickness, and then propagate along the circumference of the pipeline.

(2) The cause of fatigue failure is that the contact gap between the outer surface of the steel pipe and the inner surface of the fixture is too small. Besides, the severe roughness of the inner surface of the fixture leads to the stress concentration there, which becomes the weakest area in the system. Stress concentration can be eliminated after the inner surface of the fixture is polished.

(3) When the stress amplitude is too high, the S-N curve of the base material coincides with the $\mathrm{S}-\mathrm{N}$ curve of the welded joint. If the base material is affected by prefabricated defects, the fatigue strength of the base material in the stress range will be lower than that of the welded joint, causing the base material to crack first.

\subsection{For pipelines whose failure location is the welded joint}

(1) In general, cracks originate at welded joints and weld toes, first penetrate the wall thickness and then propagate along the circumference of the pipe, finally causing failure.

(2) The welded joints and weld toes along the fusion line are prone to discontinuities caused by microundercuts or inclusions, making these sharp defects the initial source of fatigue cracks. Therefore, the microundercut formed during the welding process will have a significant impact on the fatigue strength of the welded joint, and such defects should be strictly controlled during the welding process.

(3) For pipelines of welded joints with prefabricated cracks, the cracks will cause the local stress at the crack tip in the pipeline to increase. In this way, the fatigue fracture of the pipeline will occur at the designated prefabricated crack, which can be assessed as the designated failure of the pipeline. Studying the fracture process and fracture criterion of this type of pipeline is much closer to the actual engineering situation.

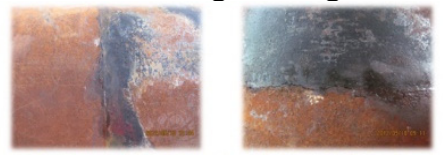

2\# Steel pipes

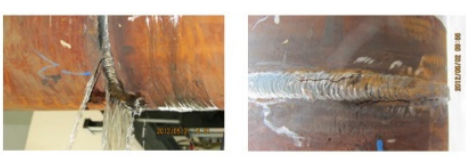

3\# Steel pipes

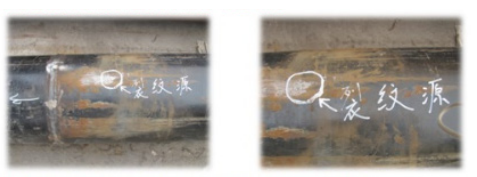

4\# Steel pipes

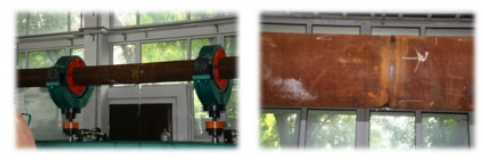

5\# Steel pipes

Figure2. Schematic diagram of pipeline failure

\section{Conclusion}

A full-scale fatigue test system and its test analysis technology applied for ZY-PFS2000 pipelines have been 
first developed in China, in which the effects of welding residual stress, stress concentration, initial welding defects, pipeline internal pressure shutdown, and internal medium fluctuations on the fatigue life of full-scale pipelines were comprehensively taken into account. On the one hand, the study in this paper is beneficial to increasing engineering personnel's understanding of the fatigue resistance of welded structures, and improving the design capabilities of pipeline structure forms and joint forms, which enables the designed welding structure to be more rational and develop higher fatigue strength while strictly controlling the welding quality. On the other hand, these technologies directly face the weakness of poor fatigue performance of welded joints, assisting in strict control of welding quality during the manufacturing process to prevent and reduce the occurrence of welding defects.

Through the full-scale fatigue test (four-point bending + internal pressure) of the X65 submarine pipeline, the fatigue cycles of different specifications of pipelines under different stress amplitudes were obtained. The fatigue failure of the pipeline can be classified into three types, including the unknown location of the pipeline failure, the failure location of the pipeline being the base material, and the failure location of the pipeline being the welded joint. Moreover, the fatigue loading results were evaluated and analyzed by the international standard of BS 7608 and DNV C203.

This paper believes that pipeline full-scale fatigue test research will be more widely used in the field of fatigue evaluation and integrity analysis of submarine pipelines in the future. Based on the currently limited data of the fullscale fatigue test of submarine pipelines and the need to further improve the fatigue test technology, this paper should focus on the following research directions when developing the full-scale fatigue test technology of submarine pipelines: (1) Optimize the selection of the best full-scale fatigue test type according to the service environment of the pipeline to achieve the coordinated development of multiple types of fatigue tests (including three-point and four-point bending fatigue, vortexinduced high-frequency vibration fatigue, and axial tensile fatigue); (2) Further improve the stress-strain testing technology and strengthen the coupling acquisition and processing capabilities of multi-channel data at the welded joints; (3) Improve the collection, processing, and analysis capabilities of full-scale fatigue test data with the help of theories including local stress and strain analysis and fracture mechanics to form a more scientific theoretical system of fatigue evaluation and analysis.

\section{Acknowledgment}

This research is supported by the special fund for the basic research business of the central public welfare research institutes (TKS 20200320, TKS190108) and 2020 key science and technology projects of transportation industry (2020-MS3-097).

\section{References}

1. J. D. Majumdar, "Underwater welding-present status and future scope," Journal of Naval Architecture and Marine Engineering, 2006, vol. 3, pp. 38-47.

2. J. Labanowski, "D. Fydrych and G. Rogalski, Underwater welding review," Advances in Materials Sciences, 2008, vol. 8 , pp. 11-22.

3. A. S. Azar and O. M. Akselsen, "Analytical Modeling of Weld Bead Shape in Dry Hyperbaric GMAW Using Ar-He Chamber Gas Mixtures," Journal of materials engineering and performance, 2013, vol. 22, pp. 673680 .

4. M. MOHIPOUR, "High Pressure Pipelines-trends for the New Millennium," Proceedings of International Pipeline Conference, ASME. 2000, pp. 32-36.

5. H. Fostervoll and R. Aune, "Remotely Controlled Hyperbaric Welding of Subsea Pipelines," Pipeline Technology Conference, 2011, pp. 34-37.

6. A. H. Varma, B. W. Russell, and B. Wallace, "Largescale rotating bending fatigue tests for offshore pipe connections," Experimental Mechanics, vol. 37, pp. 147-153, Jun 1997.

7. S.H. Hashemi and D. Mohammadyani. "Characterisation of weldment hardness, impact energy and microstructure in API X65 steel," International Journal of Pressure Vessels and Piping, 2012, vol. 98, pp. 8-15.

8. S. Shanmugam, R. D. K. Misra and J. Hrtmann. "Microstructure of high strength niobium-containing pipeline steel," Material Science and Engineering A, 2006, vol. 441, pp. 215-229.

9. SES, "fatigue testing," Stress Engineering Services INC. 2010, vol. 2010, available.

10. H. C. Fang. "Safety and reliability analysis of long distance oil and gas pipeline," Beinjing: Petroleum Industry Press, 2002.

11. H.C. Fang and G. M. Chen, "Reliability analysis of offshore structures in ice area," Beinjing: Petroleum Industry Press, 2000.

12. W. X. Yao, "Structural fatigue life analysis," Beinjing: National Defense Industry Press, 2003.

13. S. J. Zhang and D. Y. Shi, "Fatigue and fracture of offshore structures," Harbin, Harbin Engineering University Press, 2004.

14. J. Y. Pan, "Fatigue of welded structures: a review of fatigue tests at home and abroad," China Railway Science, 1983, vol. 1, pp. 73 - 84 .

15. H. Motohashi, N. Hagiwara and T. Masuda, "Tensile properties and microstructure of weld metal in MAG welded X80 pipeline steel," Welding International, 2005, vol. 19, pp. 100-108.

16. R. Song, "Overview of processing microstructure and mechanical properties of ultrafine grained bcc steels," Materials Science and Engineering: A, 2006, vol. 441, pp. 1-17.

17. Y. H. Hu, D. Y.Tang and Z. T. Fang, "Research status and development trend of full scale fatigue test technology for offshore pipeline," Petroleum Engineering Construction, 2013, vol. 39, pp. 1-6. 
18. D. Y.Tang, Z. T. Fang and Y. H. Hu, "Development of full scale fatigue testing machine for offshore pipeline," Petroleum Engineering Construction, 2013, vol. 39 , pp. 20-25.

19. J. C. Newman and I. S. Raju, "An empirical stressintensity factor equation for the surface crack," Engineering Fracture M echanics, 1981, vol. 15, pp. 185-192

20. X. B. Lin and R. A. Smith, "Numerical analysis of fatigue growth of external surface cracks in pressurized cylinders," Int.Journal of Pressure Vessels and Piping, 1997, vol. 71, pp. 293-300.

21. J. M. Stephen and G. R. Razmjoo, "Fatigue performance of large girth welded steel tubes," 17th International Conference on Offshore Mechanics and Arctic Engineering, OMAE98-23(55).

22. J. M. Stephen, B. S.Julian and G. R. Razmjoo, “An investigation of the fatigue performance of riser girth welds," 25th International Conference on Offshore Mechanics and Arctic Engineering, OMAE, 200692(315).

23. K. Hasegawa, K. Sakata and K. Miyazaki, "Fatigue strength for pipes with allowable flaws and design curve," International Journal of Pressure Vessels and Piping, 2002, vol. 79, pp. 37-44.

24. G. F. Miscow, P. E. V. de Miranda and T. A. Netto, "Techniques to characterize fatigue behavior of full size drill pipes," International Journal of Fatigue, 2004, vol. 26, pp. 575-584.

25. T. A. Netto, M. I. Lourenco and A. Botto, "Fatigue performance of pre-strained pipes with girth weld defects: Full-scale experiments and analyses," International Journal of Fatigue, 2008, vol. 30, pp. 767-778.

26. http://www.ntnu.no/. Official homepage of Norwegian University of science and technology.

27. Y.L. Li, C. J. Zhuang and Y.R. Feng, "Fatigue life analysis and prediction of oil and gas pipeline," Oil and gas storage and transportation, 2004 , vol. 23, pp. $41-43$.

28. C. J. Zhuang, "Full-scale fatigue test on X60 spiral linepipe," 25th International Conference on Offshore Mechanics and Arctic Engineering, OMAE,200692031. 